\title{
(1)
}

UNIVERSIDAD PERUANA DE CIENCIAS APLICADAS

FACULTAD DE CIENCIAS DE LA SALUD

CARRERA DE ODONTOLOGÍA

\section{DETERMINACIÓN DEL NIVEL DE RIESGO Y SU RELACIÓN COMO GUÍA PARA UN MANEJO CLÍNICO SEGÚN EL ÍNDICE BEWE EN LOS ESTUDIANTES DE 12 A 16 AÑOS DE UNA ESCUELA PÚBLICA DE CHORRILLOS}

TESIS

Para optar el título profesional de:

CIRUJANO DENTISTA

AUTOR

Angie Rosse Gallo Oropeza

(0000-0002-0334-2263)

ASESOR DE TESIS:

Dra. Leslie Caroll Casas Apayco

(0000-0001-7370-4808) 


\section{Lima, Perú}

2018

DEDICATORIA

A Dios por permitirme culminar esta etapa de mi vida.

A mis padres y hermanos quienes fueron de apoyo incondicional durante este proceso. 


\section{AGRADECIMIENTOS}

Agradezco al Señor de chocan, patrono de Querecotillo y a a Dios por permitirme culminar esta etapa de mi vida.

A mis padres por su apoyo y por brindarme todo lo necesario para salir adelante A mi asesora Leslie Casas por el conocimiento, su tiempo, su apoyo y exigencia. A mis profesores de quienes he recibido la mejor formación sobre esta carrera 


\section{RESUMEN}

Objetivo: El objetivo de este estudio es determinar el nivel de riesgo y su relación con el manejo clínico proporcionado por el índice BEWE en los estudiantes de 12 a 16 años de una escuela pública de Chorrillos.

Metodología: Se evaluaron 382 fichas obtenidas del estudio "Prevalencia de erosión dental en estudiantes de 12 a 16 años utilizando Basic Erosive Wear Examination (BEWE) en una institución educativa del distrito de Chorrillos". Se determinó el tamaño de muestra utilizando la fórmula de estimación de una proporción, tomando un nivel de confianza de 95\%, precisión de 5\% y una proporción esperada de $12.04 \%$. Para la evaluación del índice BEWE, se registró la superficie más afectada de cada sextante según la codificación de este índice- 4 niveles $(0,1,2,3)$ y se obtuvo el score acumulativo de los sextantes para determinar el nivel de riesgo y guía de tratamiento. Los datos fueron analizados mediante estadística descriptiva y se aplicó la prueba efectiva de Chi2 para relacionar el nivel de riesgo con las variables género y edad $(\alpha=0,05)$.

Resultados: El score acumulativo más prevalente fue el score 2 tanto en género femenino y masculino con $5.35 \%$ (10) y $6.15 \%$ (12) estudiantes, respectivamente. El nivel de riesgo más prevalente fue "No presenta riesgo" en género masculino con 97.44\% (190) y en femenino con 95.72\% (179). Según la edad, el score más prevalente fue el score 2 para la edad de 16 con $7.89 \%$ (6) y para la edad de 14 con $6.41 \%$ (5). En cuanto al nivel de riesgo, el rango más prevalente fue el "No presenta riesgo" en la edad de 12 con 97.59\% (81) y para la edad de 14 con $98.72 \%$ (77) seguido del riesgo "bajo" con 6.58\% (5) para la edad de 16 y con 3.80\%(3) para la edad de 13 años.

Conclusiones: El score acumulativo con mayor prevalencia fue el score 2 tanto para las variables de género y edad. El nivel de riesgo más prevalente fue el nivel de "no presenta riesgo".

Palabras clave: Riesgo, Erosión de los dientes, estudiantes 


\begin{abstract}
Objective: The aim of this study was to determine the risk level and its relation as a guide for clinical management according to the BEWE index in students from 12 to 16 years of age in a public school in Chorrillos.

Material and methods: 382 records were evaluated of the study "Prevalence of dental erosion in students aged 12 to 16 years using Basic Erosive Wear Examination (BEWE) in an public school of the Chorrillos district". The sample size was determined using the estimation formula of a proportion, confidence level $95 \%$, precision $5 \%$ and an expected proportion of $12.04 \%$. For the evaluation of the BEWE index, the most affected area of each sextant was recorded according to the coding of this index- 4 levels $(0,1,2.3)$ and the cumulative score of the sextants was obtained to determine the level of risk and treatment guide. The data were analyzed by descriptive statistics and for the relation between the level of risk with the variables gender and age, the Chi2 test was applied $(\alpha=0.05)$.
\end{abstract}

Results: The most prevalent cumulative score was score 2 in both the female and male gender with $5.35 \%(10)$ and $6.15 \%$ (12) students, respectively. The most prevalent level of risk was "No risk" in the male gender with 97.44\% (190) and in female with $95.72 \%$ (179). According to age, the most prevalent score was score 2 for age 16 with $7.89 \%$ (6) and for age 14 with $6.41 \%$ (5). Regarding the level of risk, the most prevalent range was the "No risk" at the age of 12 with $97.59 \%$ (81) and for the age of 14 with $98.72 \%$ (77) followed by the "low" risk with $6.58 \%$ (5) for the age of 16 and with 3.80 (3) for the age of 13 years.

Conclusions: The cumulative score with the highest prevalence was score 2 for both gender and age variables. The most prevalent level of risk was the level of "No risk".

Key words: Risk, Tooth erosion, Students 


\section{TABLA DE CONTENIDO}

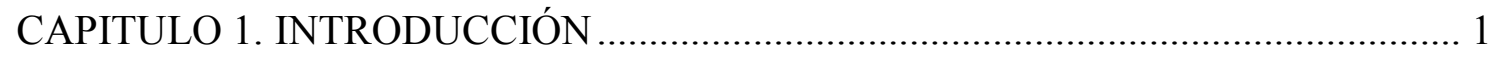

CAPÍTULO 2. PLANTEAMIENTO DE LA INVESTIGACIÓN ................................... 3

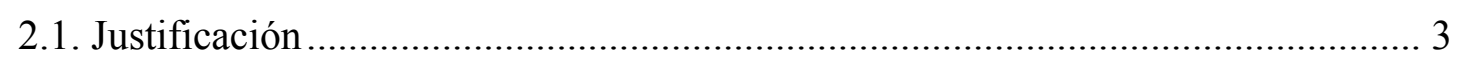

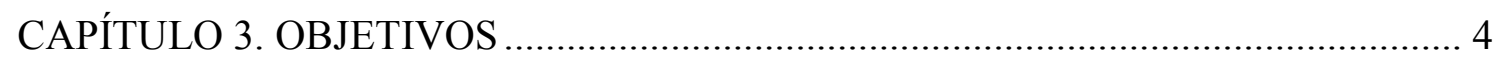

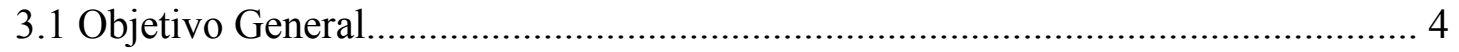

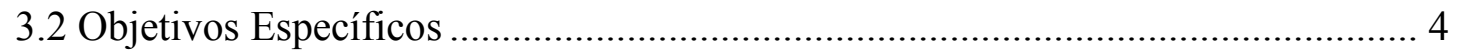

CAPÍTULO 4. MATERIALES Y MÉTODOS ............................................................. 5

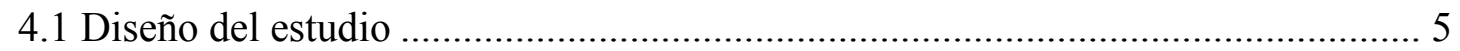

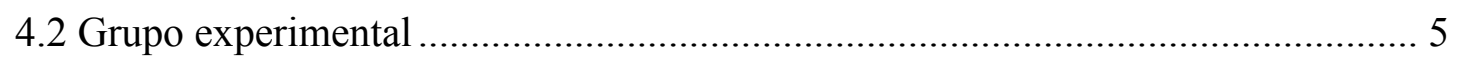

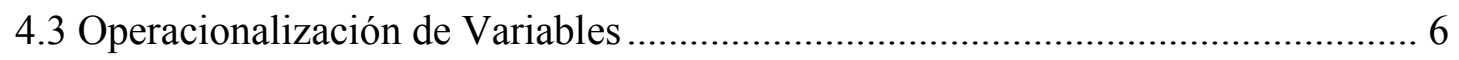

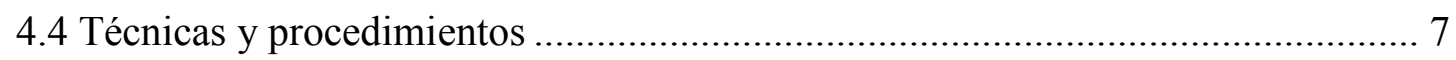

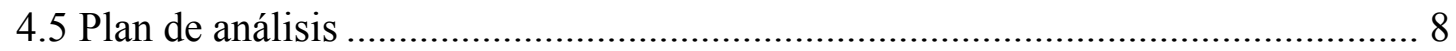

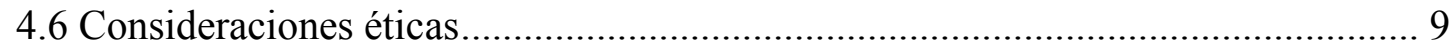

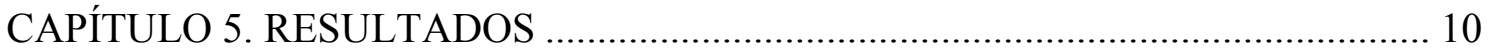

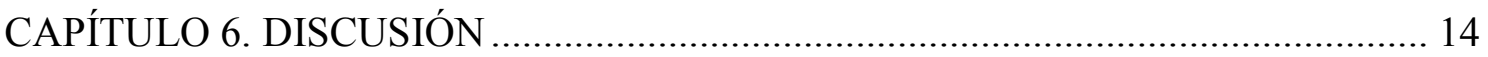

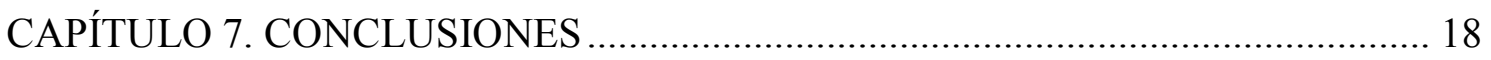

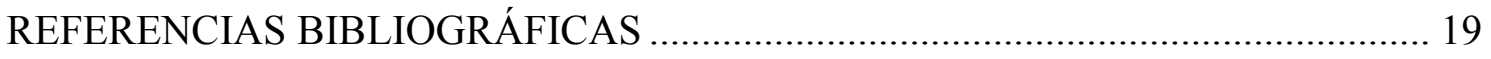

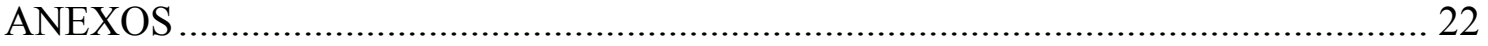




\section{ÍNDICE DE TABLAS}

TABLA N ${ }^{\circ} 1$ Distribución y correlación del score acumulativo BEWE según edad y

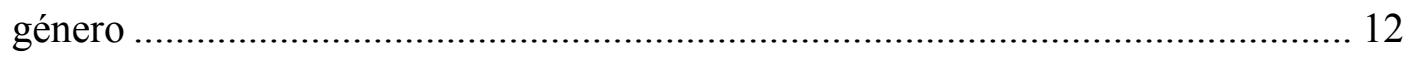

TABLA N 2 Distribución y correlación del nivel de riesgo según edad y género ........ 13 


\section{CAPITULO 1. INTRODUCCIÓN}

La erosión dental es una condición multifactorial. Esta se refiere a una pérdida de la superficie causada por ácidos extrínsecos e intrínsecos, los cuales conllevan al desarrollo de problemas estéticos y funcionales. ${ }^{(1,2,3)}$ Esta patología se manifiesta como un desgaste inicial en la superficie dental, la cual se puede considerar como el primer signo clínico, y puede llegar hasta un cambio estructural de la pieza si no se realiza un diagnóstico temprano. ${ }^{(4,5)}$ En cuanto las características clínicas, en sus primeros signos se manifiesta tornando el esmalte de una superficie mate y suave, por otro lado en las etapas más avanzadas ocurre un cambio en la morfología de la pieza dental, pérdida de la anatomía dental, lesiones socavadas en forma "cuchara" y sensibilidad por exposición dentinaria. ${ }^{(4-6,7,8)}$

El diagnóstico de las lesiones erosivas por parte del odontólogo, solo es posible cuando estas se encuentran en un estadio muy avanzado, sin embargo el diagnóstico precoz es importante para establecer tratamientos preventivos. Si bien existen diversos índices eficaces para el diagnóstico clínico del desgaste dental erosivo, la mayoría de los índices solo se limitan a la evaluación de presencia o ausencia de erosión dental, tales como los índices propuestos por Eccles en 1978, el índice de Smith y Knight en 1984, posteriormente el índice de Lussi en 1996, el índice de O'Sullivan en el 2000 y el índice Exact Tooth Wear Index (ETWI) en el 2008. ${ }^{(14-21)}$

En 2008, se introdujo un índice llamado Basic Erosive Wear Examination (BEWE) por Barlett y col. El objetivo de este índice es evaluar la severidad de la erosión dental, y registra la superficie más afectada en cada sextante con un puntaje de 4 niveles $(0,1,2$, 3). El puntaje acumulado determina un nivel de riesgo y guía para el tratamiento. ${ }^{(25-27)}$ Actualmente, este índice sirve como herramienta para estudios epidemiológicos y clínicos, y permite hallar la prevalencia de erosión dental de una población. Este índice se complementa con un cuadro de nivel de riesgo de erosión relacionado al score acumulativo obtenido. Esta guía de tratamiento incluirá la identificación y eliminación 
de los posibles factores etiológicos, realizando tratamientos preventivos o restauradores según el nivel de riesgo encontrado. ${ }^{(23,24,26,27)}$

En la actualidad, no existen estudios en Perú relacionados a la prevalencia de erosión dental y sus niveles de riesgo-guía tratamiento. En un estudio reciente, se evaluó la prevalencia de erosión dental en una escuela pública de Lima-Perú donde se halló una prevalencia del $12.04 \%$ en el rango de edad de $12-16$ años de edad. ${ }^{(26)}$ Por tal motivo, el objetivo del estudio es determinar el nivel de riesgo y su relación como guía para un manejo clínico según el índice BEWE en los estudiantes de 12 a 16 años de una escuela pública de Chorrillos. 


\section{CAPÍTULO 2. PLANTEAMIENTO DE LA INVESTIGACIÓN}

\subsection{Justificación}

El presente estudio tiene importancia teórica, ya que proporcionara información sobre el manejo clínico según el nivel de riesgo obtenido de la prevalencia de erosión dental en estudiantes de 12 a 16 años de edad en la Institución Educativa Pública "María Auxiliadora" del distrito de Chorrillos, mediante el índice de BEWE.

Por otro lado, tiene importancia clínica, ya que proporcionará información sobre las secuencias de tratamiento tales como la observación, prevención y restauración relacionadas al manejo clínico de acuerdo a cada nivel.

Así mismo, revela una importancia social, ya que en el Perú en este rango de edad no existe suficiente información científica sobre los posibles niveles de riesgo establecidos por un índice validado

El propósito del presente estudio determinar el nivel de riesgo y su relación como guía para un manejo clínico según el índice BEWE en los estudiantes de 12 a 16 años de una escuela pública "María Auxiliadora". 


\section{CAPÍTULO 3. OBJETIVOS}

\subsection{Objetivo General}

Determinar el nivel de riesgo de erosión dental y el manejo clínico según el índice BEWE obtenido de la prevalencia en estudiantes de 12 a 16 años en la Institución Educativa

Pública "María Auxiliadora" del distrito de Chorrillos.

\subsection{Objetivos Específicos}

1. Determinar el score acumulativo según la prevalencia obtenida según el índice BEWE en estudiantes de 12 a 16 años en la Institución Educativa Pública "María Auxiliadora" del distrito de Chorrillos según edad y género.

2. Determinar el nivel de riesgo según el índice BEWE en estudiantes de 12 a 16 años en la Institución Educativa Pública "María Auxiliadora” según edad y género.

3. Determinar la correlación entre el nivel de riesgo y manejo clínico según el índice BEWE según edad y género. 


\section{CAPÍTULO 4. MATERIALES Y MÉTODOS}

\subsection{Diseño del estudio}

El presente estudio será de tipo Analítico-Descriptivo.

\subsection{Población y muestra}

La población está conformada por estudiantes de 12 a 16 años matriculados en la Institución Educativa Pública "María Auxiliadora" de Chorrillos, Lima-Perú, en el periodo del año escolar de 2016. ${ }^{(26)}$

La muestra está constituida por 382 fichas de recolección de datos del estudio realizado previamente denominado "Prevalencia de erosión dental en estudiantes de 12 a 16 años utilizando Basic Erosive Wear Examination (BEWE) en una institución educativa del distrito de Chorrillos". Esto se determinó mediante el software estadístico Epidat ${ }^{\circledR}$ versión 4.2, utilizando la fórmula de estimación de proporción, para lo cual se tomaron los datos de proporción esperada $(12.06 \%)$ de la investigación previamente indicada realizada por Hayakawa y col. ${ }^{(26)}$ Esta fórmula determino un tamaño de 163 fichas, pero para fines de este estudio se utilizara el total de las fichas recolectadas ( $n=382)$. Además se tomó un valor de 95\% para el nivel de confianza y 5\% de precisión.

\section{Criterios de Selección}

\section{Criterios de inclusión}

1. Fichas de datos de estudiantes que hayan firmado el asentimiento informado y consentimiento informado.

2. Fichas de datos que tengan los datos completos de género, edad y score acumulativo registrado. 


\subsection{Operacionalización de Variables}

\begin{tabular}{|c|c|c|c|c|c|}
\hline Variable & $\begin{array}{l}\text { Definición } \\
\text { operacional }\end{array}$ & Indicadores & Tipo & $\begin{array}{l}\text { Escala de } \\
\text { medición }\end{array}$ & Valores \\
\hline Género & $\begin{array}{l}\text { Grupo al que } \\
\text { pertenecen los } \\
\text { seres humanos } \\
\text { de cada sexo }\end{array}$ & DNI & Cualitativo & $\begin{array}{c}\text { Nominal } \\
\text { Dicotómico }\end{array}$ & $\begin{array}{l}\text {-Femenino } \\
\text {-Masculino }\end{array}$ \\
\hline Edad & $\begin{array}{l}\text { Tiempo que ha } \\
\text { vivido una } \\
\text { persona desde } \\
\text { su nacimiento }\end{array}$ & DNI & Cuantitativo & $\begin{array}{l}\text { Razón } \\
\text { Politómica }\end{array}$ & $12,13,14,15,16$ \\
\hline $\begin{array}{c}\text { Score } \\
\text { acumulativo }\end{array}$ & $\begin{array}{l}\text { Nro de la suma } \\
\text { de los } \\
\text { sextantes que } \\
\text { guiara a un } \\
\text { nivel de riesgo }\end{array}$ & $\begin{array}{l}\text { Ficha de } \\
\text { recolección } \\
\text { de datos }\end{array}$ & Cuantitativo & $\begin{array}{l}\text { Razón } \\
\text { Politómica }\end{array}$ & $\begin{array}{c}1,2,3,4,5,6,7,8,9,10,11,12,13 \\
14\end{array}$ \\
\hline $\begin{array}{l}\text { Nivel de } \\
\text { Riesgo }\end{array}$ & $\begin{array}{c}\text { Grado de } \\
\text { riesgo según } \\
\text { índice BEWE }\end{array}$ & $\begin{array}{l}\text { Ficha de } \\
\text { recolección } \\
\text { de datos }\end{array}$ & Cualitativo & $\begin{array}{l}\text { Ordinal } \\
\text { Politómica }\end{array}$ & $\begin{array}{c}\text {-No hay riesgo }(0-2) \\
\text {-Bajo }(3-9) \\
\text {-Moderado }(9-13) \\
\text {-Alto }(14 \mathrm{a}+)\end{array}$ \\
\hline $\begin{array}{c}\text { Manejo } \\
\text { Clínico } \\
\text { según BEWE }\end{array}$ & $\begin{array}{l}\text { Tratamiento } \\
\text { que indica el } \\
\text { índice de } \\
\text { acuerdo al } \\
\text { nivel de riesgo }\end{array}$ & $\begin{array}{l}\text { índice } \\
\text { BEWE }\end{array}$ & Cualitativo & $\begin{array}{l}\text { Ordinal } \\
\text { Politómica }\end{array}$ & $\begin{array}{l}\text {-No hay riesgo: observación y } \\
\text { mantenimiento en intervalos de } 3 \text { años } \\
\text {-Bajo: evaluación de la dieta, higiene } \\
\text { oral, mantenimiento, observación en } \\
\text { intervalos de } 2 \text { años } \\
\text {-Moderado: evaluación de la dieta, } \\
\text { higiene oral, identificación de factores } \\
\text { etiológicos, estrategias para disminuir } \\
\text { impactos y monitoreo en intervalos de } \\
\text { 6-12 meses. } \\
\text {-Alto: evaluación de la dieta, higiene } \\
\text { oral, identificación de factores }\end{array}$ \\
\hline
\end{tabular}




\subsection{Técnicas y procedimientos}

\section{Base de datos}

El investigador se encargará del análisis de la base de datos de la investigación "Prevalencia de erosión dental en estudiantes de 12 a 16 años utilizando Basic Erosive Wear Examination (BEWE) en la Institución Educativa Pública "María Auxiliadora "del distrito de Chorrillos. ${ }^{(26)}$ Este estudio consistió en evaluar la presencia de erosión dental según edad, género y localización de los dientes permanentes en 382 estudiantes.

\section{Proceso de información}

Se seleccionaran las fichas de datos que cumplan que los criterios de inclusión y exclusión.

\section{Registro del Score acumulativo BEWE}

Según el índice BEWE, el score acumulativo registra el valor más alto de cada sextante (0- cuando no haya presencia de erosión dental, 1 - cuando la pieza presente pérdida inicial de la superficie dental, 2 - cuando haya una pérdida menor $<50 \%$ de la superficie dental y 3- cuando haya una pérdida mayor $>50 \%$ de la superficie dental) y posteriormente, se suman los valores de los 6 sextantes y el puntaje acumulado determinará el score BEWE acumulativo. $^{(27)}$ (Anexo 2)

\section{Obtención del nivel de riesgo y manejo clínico}

Una vez obtenido el score acumulativo de cada ficha de datos, este valor numérico obtenido será comparado con la tabla de nivel de riesgo BEWE: No riesgo (0-2), Bajo (38), Moderado (9-13) y Severo (14 a más). ${ }^{(15)}$ De acuerdo a este nivel de riesgo, el índice BEWE lo relaciona con una guía para el manejo clínico.(Anexo 3) 
Si no existe riesgo, el manejo clínico será observación y mantenimiento de rutina repetido en un intervalo de 3 años.

Si existe riesgo bajo, el manejo clínico será la evaluación de la dieta, higiene oral, asesoría, observación y mantenimiento de rutina. Repetir en intervalos de 2 años.

Si existe riesgo medio, el manejo clínico será la evaluación de la dieta, higiene oral, asesoría, identificar los factores etiológicos principales para la perdida de tejidos y desarrollar estrategias para eliminar los respectivos impactos. Considerar medidas de fluorización y otras estrategias para incrementar la resistencia de las superficies dentarias. Idealmente, evitar la colocación de restauraciones y monitorear la erosión dental con estudios, fotografías o impresiones. Repetir en intervalos de 6-12 meses.

Si existe riesgo alto, el manejo clínico será la evaluación de la dieta, higiene oral, asesoría, identificar los factores etiológicos principales para la perdida de tejidos y desarrollar estrategias para eliminar los respectivos impactos. Considerar medidas de fluorización y otras estrategias para incrementar la resistencia de las superficies dentarias. Idealmente, evitar la colocación de restauraciones y monitorear la erosión dental con estudios, fotografías o impresiones. Especialmente en casos de progresión severa considerar cuidado especial, ya que puede involucrar restauraciones. Repetir 6-12 meses. ${ }^{(27)}$

Se procesarán los datos para obtener el score acumulativo, el nivel de riesgo- manejo clínico de acuerdo al índice BEWE según el género y edad en una ficha de datos específica para este estudio.

\subsection{Plan de análisis}

Para el análisis univariado, se procedió a obtener la estadística descriptiva, frecuencia absoluta y relativa de las variables score acumulativo y nivel de riesgo según género y edad. 
Para el análisis bivariado se procedió a utilizar la prueba de Chi cuadrado para relacionar score acumulativo y el nivel de riesgo según género y edad $(\mathrm{p}<0.05)$. La base de datos se realizó en el programa Microsoft Excel y se analizaron los resultados mediante los paquetes estadísticos $\operatorname{Stata}^{\circledR}$ versión 13.0.

\subsection{Consideraciones éticas}

Este estudio no presenta implicaciones éticas debido a que se tomaran los datos de la base principal del estudio realizado "Prevalencia de erosión dental en estudiantes de 12 a 16 años utilizando Basic Erosive Wear Examination (BEWE) en una

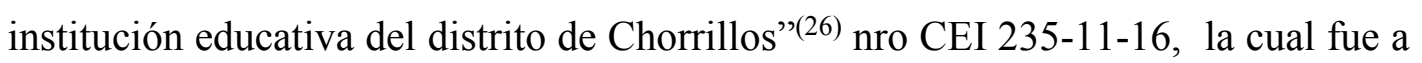
partir de una revisión no invasiva de las superficies dentales y se registró la información en una ficha para determinar su grado de severidad según el índice de BEWE. 


\section{CAPÍTULO 5. RESULTADOS}

Del total de 382 fichas evaluadas, según el índice de BEWE, el score acumulativo 0 fue el más prevalente con 89.23\% (174) para el sexo masculino; seguido del score 2 con $12 \%(6.15)$, y por último, el score 6 con $0.51 \%$ (1). Por otro lado, para el sexo femenino, el score acumulativo más prevalente fue el 0 con $86.63 \%$ (162), seguido del score 2 con $10 \%$ (5.35), y el score 5 con $0.51 \%$ (1). (Tabla 1)

Según el rango de edad, los niños de 12 años con score de 0 presentaron la mayor prevalencia de 87.95\%(73), con score 2 de 4.82\%(4). Los de 13 años con score 0 presentaron una prevalencia de $86.08 \%(68)$, con score 1 de $6.33 \%(5)$ y con score 2 de $2.53 \%(2)$. Los de 14 años con score 0 presentaron una prevalencia de $92.31 \%(72)$ y con score 2 de 6.41\%(5). Los de 15 años con score 0 presentaron una prevalencia de $90.91 \%(60)$ y con score 2 de 6.06\%(4). Finalmente, los de 16 años con score 0 presentaron una prevalencia de $82.89 \%$ (63) y con score 2 de $7.89 \%(6)$. Los valores de "score acumulativo" restantes fueron de 3, 4, 5 y 6 en menor cantidad respectivamente. No se encontraron diferencias significativas entre el score acumulativo entre género y edad. (Tabla 1)

Con respecto al nivel de riesgo, el sexo masculino presentó una mayor prevalencia en el rango de 0-2 ("no hay riesgo") con 97.44\% (190) y en el rango de 3-9 ("riesgo bajo") una prevalencia de $2.56 \%$ (5). El sexo femenino presentó una prevalencia en el rango de 0-2 ("no hay riesgo") con 95.72\% (179) y en el rango de 3-9 ("riesgo bajo") una prevalencia de $4.28 \%$ (8). (Tabla 2)

La edad más prevalente en el rango de 0-2, fue la de 12 años con 97.59\% (81), seguido de la edad de 14 años con 98.72\% (77), la edad de 13 años con 96.20\% (76), la edad de 16 años con 93.42\% (71); por último, la edad de 15 años con 96.97\% (64). Por otro lado, en el rango de 3-9, la edad con mayor prevalencia fue la de 16 años con 6.58\% (5), seguido de la edad de 13 años con 3.80\% (3), la edad de 15 años con 3.03\% (2), la edad 
de 12 años con $2.41 \%$ (2); por último, la edad de 14 años con $1.28 \%$ (1). Según los resultados obtenidos, no existen diferencias estadísticamente significativas entre el nivel de riesgo con género y edad. (Tabla 2) 


\section{TABLA 1}

Distribución y correlación del score acumulativo BEWE según edad y género

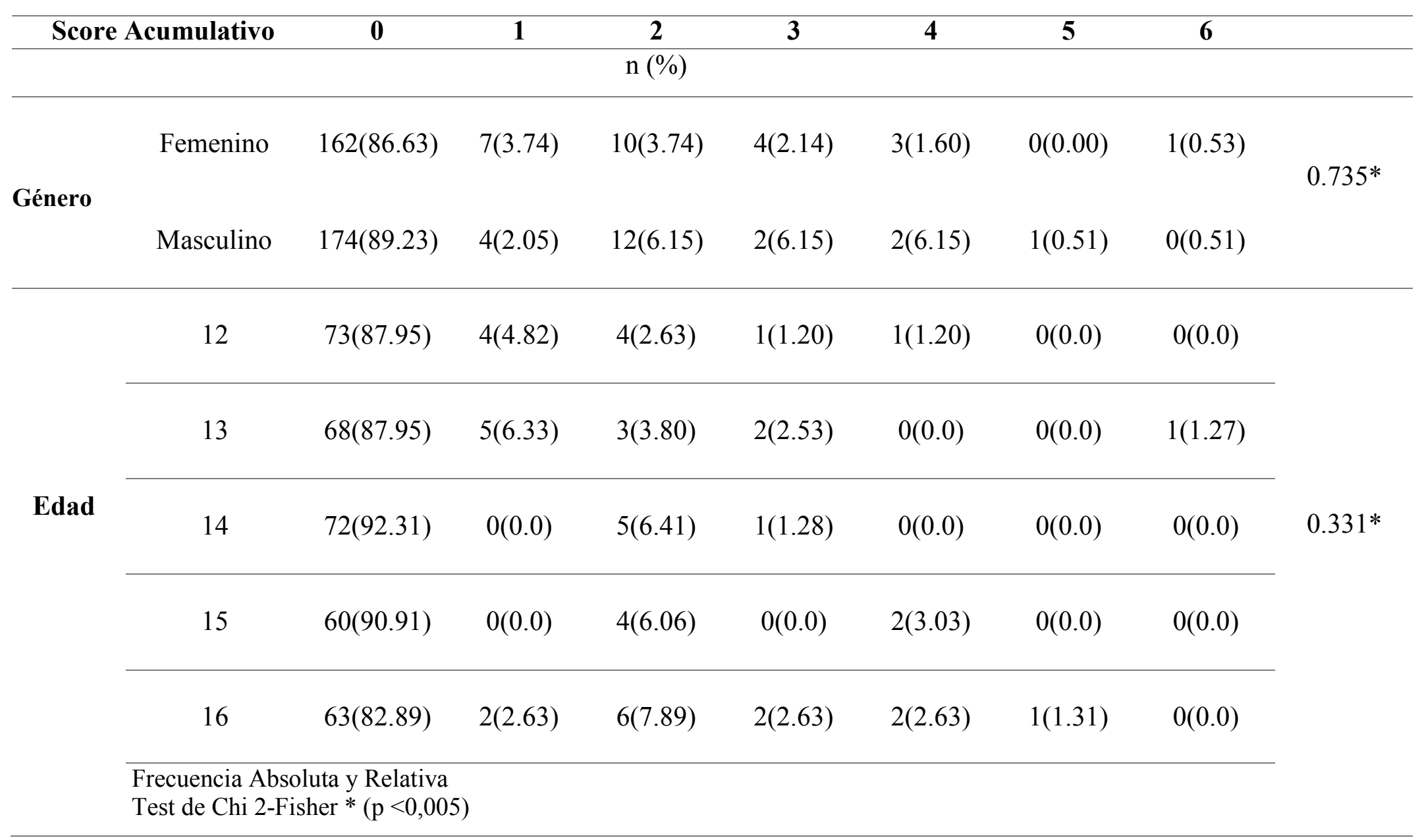


TABLA 2

Distribución y correlación del nivel de riesgo según edad y género

\begin{tabular}{|c|c|c|c|c|c|c|}
\hline \multicolumn{2}{|c|}{$\begin{array}{c}\text { Nivel de riesgo } \\
\text { BEWE }\end{array}$} & $\begin{array}{c}\text { No presenta } \\
\text { riesgo } \\
(0-2)\end{array}$ & $\begin{array}{l}\text { Bajo } \\
(3-8)\end{array}$ & $\begin{array}{c}\text { Mediano } \\
(9-13)\end{array}$ & \multirow[t]{2}{*}{$\begin{array}{c}\text { Alto } \\
\left(14 \mathrm{a}^{+}\right)\end{array}$} & \multirow{4}{*}{$0.85^{*}$} \\
\hline \multicolumn{5}{|c|}{$\mathrm{n}(\%)$} & & \\
\hline \multirow{2}{*}{ Género } & Femenino & $179(95.72)$ & $8(4.28)$ & $0(0)$ & $0(0)$ & \\
\hline & Masculino & $190(97.44)$ & $5(2.56)$ & $0(0)$ & $0(0)$ & \\
\hline \multirow{5}{*}{ Edad } & 12 años & 81 (97.59) & $2(2.41)$ & $0(0)$ & $0(0)$ & \multirow{5}{*}{$0.491 * *$} \\
\hline & 13 años & $76(96.20)$ & $3(3.80)$ & $0(0)$ & $0(0)$ & \\
\hline & 14 años & 77 (98.72) & $1(1.28)$ & $0(0)$ & $0(0)$ & \\
\hline & 15 años & 64 (96.97) & $2(3.03)$ & $0(0)$ & $0(0)$ & \\
\hline & 16 años & $71(93.42)$ & $5(6.58)$ & $0(0)$ & $0(0)$ & \\
\hline
\end{tabular}

Frecuencia absoluta y relativa

Test de Chi2 * Fisher ** $(p<0,005)$ 


\section{CAPÍTULO 6. DISCUSIÓN}

El objetivo de este estudio fue determinar el nivel de riesgo de erosión dental según BEWE y su relación como guía de manejo clínico. Así mismo, evaluar si existía asociación entre las variables género y edad con el nivel de riesgo. Se encontró una mayor prevalencia del score acumulativo 2. El nivel de riesgo de erosión dental con mayor prevalencia fue "no presenta riesgo" y seguido del nivel de riesgo "bajo" en la población estudiada.

Diversos índices son utilizados para la evaluación de erosión dental. Entre los más utilizados son los índices Smith and Knight (1984), O’Sullivan (2000) y Bartlett y col (2008). ${ }^{(19-21,27)}$ El índice de Basic Erosive Wear Examination (BEWE) (2008) frente a otros índices está diseñado para registrar la incidencia de erosión dental y su manejo clínico según distintos niveles de riesgo obtenidos del score acumulativo y propone distintas estrategias de abordaje tanto preventivas como terapéuticas para cada paciente ${ }^{(27)}$ Siendo así, es necesario establecer el nivel de riesgo de cada paciente y un posible plan de tratamiento sea preventivo o restaurador.

Los resultados de este estudio revelaron que el score acumulativo con mayor distribución en el cuanto el género femenino y masculino, excluyendo al 0, fue el score 2 con 10 (5.35) \% y $12(6.15 \%)$, respectivamente. Sin embargo, estos resultados son diferentes frente a otros estudios realizados donde se aprecia una mayor incidencia de erosión dental en el género masculino. No obstante, no existen factores predisponentes que asocien el género y el rango del score acumulativo BEWE. ${ }^{(25,29,32)}$

En cuanto a la edad, el score más prevalente, fue el score 2 con $7.89 \%(6)$ para la edad de 16 años, 6.41\%(5) para la edad de 14 años, 6.06\%(4) para la edad de 15 años, 4.82\%(4) para la edad de 12 años y 3.80\%(3) para la edad de 13 años. Ningún estudiante presentó 
scores acumulativos entre 7 y 14. No existen estudios en los cuales la erosión dental presente mayor frecuencia en una edad específica. Sin embargo, el aumento de la erosión dental puede darse por el continuo consumo de bebidas carbonatadas o de la dieta que pueden presentar los estudiantes desde la erupción de los dientes. ${ }^{(24,32)}$

El nivel de riesgo más prevalente en cuanto género y edad, fue "No presenta riesgo", y en menor porcentaje el nivel de riesgo presentado en ambas variables fue el riesgo "Bajo". Los niveles de riesgo "Mediano" y "Alto" no presentaron valores significativos. Por eso, se debe hacer énfasis en la parte preventiva. Según Lussi, se debe enfatizar la importancia de realizar un diagnóstico temprano del desgaste erosivo para realizar monitorear e intervenir adecuadamente al paciente sea en la parte preventiva o restauradora. ${ }^{(4,5)}$

Actualmente son pocos los estudios en Latinoamérica enfocados en establecer el nivel de riesgo de erosión dental usando el índice de BEWE y su relación como guía de manejo clínico en el rango de edad de 12 a 16 años.

Realizar el diagnóstico e identificar la etiología de la erosión dental es importante para prevenir la aparición de esta o detener la progresión de las lesiones ya existentes. ${ }^{(4,5,7)}$ Así mismo, un factor importante para la prevención es la reducción a la exposición ácida, ya que la frecuencia y la duración del ácido pueden ser variables para el desarrollo de las lesiones erosivas. Una mayor adherencia ácida tiene un contacto más largo en la superficie de la estructura dental. ${ }^{(7,14)}$ En la actualidad, se utilizan distintos métodos preventivos, tales como el uso de enjuagues bucales y control de la dieta que consuma el paciente. Así mismo, el uso de flúor, como el flúor gel, fluoruro de estaño, barniz de flúor, fluoruro de fosfato acidificado, que han sido probados con eficacia contra la erosión dental. Según Wiegan y col, estas forman un revestimiento sobre la pieza dentaria que actúa como una barrera física ante los ácidos, además de liberar calcio y flúor. ${ }^{(17,23)}$ También, el uso de tetrafluoruro de titanio $\left(\mathrm{TiF}_{4}\right)$ es eficaz para la prevención del desgaste, debido a su interacción con el tejido dental. No obstante, la eficacia del $\mathrm{TiF}_{4}$ depende del $\mathrm{pH}$ bajo y un especial cuidado en su aplicación. ${ }^{29,30}$ Según Mundorff y col. la acción del fluoruro de titanio se debe a la capacidad de reaccionar con los átomos de oxígeno de los grupos de fosfato de la estructura dental formando óxidos de titanio estables o complejos organometálicos que 
actúan como una barrera. ${ }^{(39,40)}$ Existen pastas dentales anti-erosivos que contienen nitrato de potasio $\left(\mathrm{KNO}_{3}\right.$ ) (agente-hipersensibilidad), hidroxiapatita (HA) y otros agentes remineralizantes que han sido probados y han obtenido efectos positivos en prevención y también se recomienda el uso de pasta con flúor de 5000 ppm dos veces al día. ${ }^{(17)}$ Otros agentes como la clorhexidina, el extracto de té verde y un aumento de consumo de productos lácteos han sido probados con resultados prometedores en casos de erosión de dentina y esmalte. ${ }^{(38)}$ Así mismo, el organismo humano tiene una forma propia de protección mediante el fluido salival. Este posee propiedades físico-químicas, neutralizando los ácidos por medio del $\mathrm{pH}$ y promueve el intercambio de iones de calcio y fosfato. ${ }^{(31)}$ La saliva forma una película adquirida, que protege al diente de los ataques erosivos, ya que está compuesta por unas capas de proteínas de aminoácidos que cumplen una función de permeabilidad en la estructura dental, la cual ha demostrado su eficacia en varios estudios. ${ }^{(31,32,38,39)}$ Según Picchi y col. el bajo flujo salival y la poca capacidad del efecto buffer de la saliva pueden incrementar la erosión dental. ${ }^{(7)}$ Por eso, en casos de xerostomía donde los sustitutos de saliva son necesarios, estos deben presentar calcio y fosfato para obtener una adecuada remineralización para así contrarrestar la acción erosiva. ${ }^{(33)}$

Si hay presencia de desgaste dental erosivo, debe realizarse un tratamiento adecuado, según la severidad del caso clínico. Estos involucran el control de la sensibilidad, mejorar la función y estética, y aplicar métodos preventivos. ${ }^{(7)}$ Las medidas de higiene oral, el tiempo del cepillado de dientes y la fuerza aplicada pueden agravar las lesiones. Por eso, se aconseja realizar el cepillado dental antes de un ataque ácido o después de 30 minutos a 1 hora. ${ }^{(21,32)}$ Así mismo, se recomienda el uso de cepillo de cerdas suaves con una pasta dental con bajo nivel de abrasividad y con bajo $\mathrm{pH}$ para este tipo de pacientes. ${ }^{(32)}$ Según Bartlett D y col. las restauraciones solo deben ser realizadas en casos severos, cuando haya una pérdida de función y estética. Los materiales restauradores pueden ser resinas compuestas, cementos de ionómeros modificados con resina y restauraciones indirectas como las inscrustaciones y coronas. Se debe informar al paciente que estas restauraciones no cumplirán su función si no existe un cambio en el factor etiológico. ${ }^{(7,23)}$ 
El diagnóstico temprano podría prevenir o detener la progresión de las lesiones erosivas en los pacientes desde temprana edad. Las medida preventivas son importantes para una intervención temprana de la erosión, por eso un diagnóstico precoz es importante durante la rutina clínica. 


\section{CAPÍTULO 7. CONCLUSIONES}

1. El score acumulativo con mayor prevalencia fue el score 2 tanto para las variables de género y edad.

2. El nivel de riesgo más prevalente fue el nivel de "No presenta riesgo"seguido del nivel de riesgo "Bajo".

3. La deteccion precoz de lesiones erosivas permite la aplicación de tratamientos preventivos $\mathrm{y} / \mathrm{o}$ restauradores. 


\section{REFERENCIAS BIBLIOGRÁFICAS}

1. Álvarez C, Grille C. Revisión de la literatura: lesiones cervicales no cariogènicas. Cient Dent. 2008; 5(3):215-24.

2. Infeld T. Dental erosion. Definition, classification and links. Eur J Oral Sci. 1996; 104: $151-5$.

3. Ganss C. Definition of Erosion and Links to Tooth Wear. Monogr Oral Sci. 2006;20(2):9-16.

4. Ganss C, Lussi A. Diagnosis of erosive tooth wear. Monogr Oral Sci. 2006; $20: 32-43$.

5. Lussi A, Jaeggi T. Erosion-diagnosis and risk factors. Clin Oral Investig. 2008;12:5-13.

6. Fajardo M, Mafla A. Diagnóstico y epidemiología de erosión dental. Salud UIS. 2011; 43(2): 179-89.

7. Picchi L, Aranda P, Martines B, Magalhaes A. Dental erosion: an overview on definition, prevalence, diagnosis and therapy. Braz Dent Sci.2013; 16(1): 06-17.

8. Almeida JS, Baratieri LN, Araujo E, Widmer N. Dental erosion: understanding this pervasive condition. J Esthet Restor Dent. 2011; 23(4): 205-16.

9. Lussi A. Erosive Tooth wear- A multifactorial condition of growing concern and increasing knowledge. Monogr Oral Sci. 2006;20: 1-8.

10. Lussi A, Schaffner M. Progression of and risk factors for dental erosion and wedge-shaped defects over a 6-year period. Caries Res. 2000;34:182-187.

11. Alaraudanjoki V, Laitala M, Tjäderhane L, Pesonen P, Lussi A, Anttonen V. Association of erosive tooth wear and dental caries in northern finland birth cohort 1996-an epidemiological crooss-sectional study. BMC Oral Health. 2017;17(6):17.

12. Salas M, Nascimiento G, Hyusmans M, Demarco F. Estimated prevalence of erosive tooth wear in permanent teeth of children and adolescents: An epidemiological systematic review and meta regression analysis. J Dent.2015; $43: 42-50$.

13. Lussi A, Schaffner M, Jaeggi T. Dental erosion-diagnosis and prevention in children and adults. Int Dent J. 2007; 57:385-398. 
14. Johansson A, Ridwaan O, Gunnar C, Johansson A. Dental erosion and its growing importance in clinical practice: From Past to Present. Int J Dent. 2011; 12: 1-17.

15. Schlueter N, Hara A, Shellis R, Ganss C. Methods for the Measurement and Characterization of Erosion in Enamel and Dentine. Caries Res. 2011;45(1):1323.

16. Fang Y. Dental Erosion: Etiology, Diagnosis and prevention. Rdhmag. 2011;7680.

17. Caltrava L. Indices epidemiológicos del desgaste dental erosivo. Rodby. 2015; $4(2): 32-8$.

18. Abhinav S, Shweta K , Jay Chamele, Aditi J , Puneet G y col. Indices for measuring dental erosion. CJHS. 2013; 1(1): 52-6.

19. Ganss C, Lussi A. Current erosion indices-flawed or valid?. Clin Oral Invest. 2008; 12(1):1-3.

20. López F, Castellanos L, Martìn J, Llamas J, Segura J. Clinical measurement of tooth wear: Tooth Wear Indices. J Clin Exp Dent. 2012;4(1):48-53.

21. Fares J, et al. A New Index of Tooth Wear. Caries Res .2009; 43:119-125.

22. Lussi A, Hellwig E. Risk Assessment and preventive measures. Monogr Oral Sci. 2006; 20(12):190-199.

23. Jaeggi T, Gruninger A, Lussi A. Restorative Therapy of Erosion. Monogr Oral Sci. 2006; 20 (12):200-214.

24. Dugmore C, Rock W. The prevalence of tooth erosion in 12-year old children. $\mathrm{Br}$ Dent J 2004;196(5):279-82.

25. Mantonanaki M, Koletsi-Kounari H, Mamai-Homata E, Papaioannou W.Dental erosion prevalence and associated risk indicators among preschool children in Athens, Greece. Clin Oral Invest 2013;17:585-93

26. Kumar S, Acharya S, Mishra P, Debnath N, Vasthare R. Prevalence and risk factors for dental erosion among 11-to 14-year-old school children in South India. J Oral Sci 2013;55(4):329-36.

27. Zhang S, Chau A, Lo E, Chu CH. Dental caries and erosion status of 12-yearold 28. Hong Kong Children. BMC Public Health 2014;14:1-7

29. Baltuano K, Flores K, Farfán M, Casas L. Prevalencia de erosión dental en niños de 6 a 12 años utilizando el índice Basic Erosive Wear Examination(BEWE). ALOP. 2016; 6(1):17-22 
30. Hayakawa L. Prevalencia de erosión dental en estudiantes de 12 a 16 años utilizando Basic Erosive Wear Examination (BEWE) en una institución educativa del distrito de chorrillos. Lima: Universidad Peruana de Ciencias Aplicadas; 2017.

31. Bartlett D, Ganss C, Lussi A. Basic Erosive Wear Examination (BEWE): a new scoring system for scientific and clinical needs. Clin Oral Invest. 2008; 12(1):658.

32. Peres K y col. Dental erosion in 12- year-old schoolchildren: a cross-sectional study in southern Brazil. Int J Paediatr Dent.2005; 15: 249-55.

33. Levy F, Rios D, Buzalaf A, Magalhães C. Efficacy of TiF4 and NaF varnish and solution: a randomized in situ study on enamel erosive-abrasive wear. Clin Oral Investig. 2014; 18(4):1097-102.

34. Comar P, Cardoso A, Charone S, Grizzo T, Buzalaf A, Magalhães C. TiF4 and $\mathrm{NaF}$ varnishes as anti-erosive agents on enamel and dentine erosion progression in vitro. J Appl Oral Sci. 2015; 23(1):14-8.

35. Amaechi B, Higham S. Eroded lesions remineralisation by saliva as a possible factor in the site - specificity of human dental erosion. Arch Oral Biol 2001;46:697-703

36. Amaechi B, Highman S. Dental Erosion: possible approaches to prevention and control. J Dent. 2005; 33:243-252

37. Magalhães A, Wiegand A, Rios D, Marques H, Rabelo M. Insights into preventive measures for dental erosion. J Appl Oral Sci. 2009; 17(2): 75-86.

38. Skjorland KK, Rykke M, Sonju T. Rate of pellicle formation in vivo. Acta Odontol Scand. 1995; 53: 358-362.

39. Gutiérrez D, Isassi H, Oliver R, Padilla J, Trejo E, Huitzil E. Prevalencia de erosión dental en escolares de Tampico, Madero, Altamira y su relación con el pH salival. ALOP. 2011;1(2):170-177.

40. Mundorff SA, Little MF, Bibby BG. Enamel dissolution: II. Action of titanium tetrafluoride. J Dent Res 1972;51:1567-1571

41. Fonseca T, Pedroso C, Monteiro T, Viera C, Suzuki L, Moreira de Freitas P. The In situ Effect of Titanium Tetrafluoride Gel on Erosion/Abrasion progression in Human Dentin.

Braz

Dent

J.2017;28(3):

337-345.. 


\section{ANEXOS}

\section{Anexo 1 \\ Carta de aprobación del comité de ética}

FCS/031-05-18

Chorrillos, 18 de mayo de 2018

Alumnos

Angie Gallo Oropeza

Alumna de la Carrera de Odontología

Universidad Peruana de Ciencias Aplicadas

Presente.-

PI025-18 Determinación del nivel de riesgo y su relación como guía para un manejo clínico según el índice BEWE en los estudiantes de 12 a 16 años de una escuela pública de Chorrillos.

Estimado(a) investigador(a):

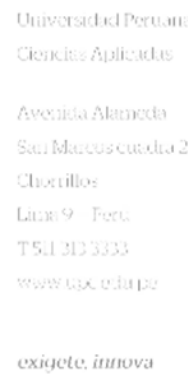

Hemos recibido el protocolo de investigación, y los documentos de soporte, los cuales han sido revisados en detalle. Luego de esta revisión, el Comité de Ética e Investigación (CEI) de la Facultad de Ciencias de la Salud ha determinado que este proyecto está APROBADO y pueden proceder con su ejecución.

Los investigadores deben de informar al Comité sobre cualquier cambio en el protocolo posterior a este dictamen. Del mismo modo, de forma anual y desde esta fecha, los investigadores deben enviar un breve informe de avances al Comité y un breve informe final al momento del cierre definitivo del estudio. Del mismo modo, ante la aparición de cualquier evento o efecto -previsible o no- que comprometa la integridad y bienestar de las unidades de estudio, los investigadores o a su equipo de investigación durante el curso de la implementación, estos deben de ser también informados inmediatamente a este comité. El comité se reserva el derecho de supervisar de manera inopinada la progresión de la investigación en cualquier momento y bajo cualquier modalidad. Nos permitimos recordar a los investigadores que la ejecución de un proyecto de investigación sin una aprobación ética vigente es una grave falta la cual puede ser sancionada con el cierre definitivo del estudio e imposibilidad de utilizar cualquier dato recolectado o generado en el mismo.

Esta aprobación tiene una duración de 18 meses a partir de la fecha de esta carta, la que puede ser renovada de ser requerido por los investigadores.

Sin otro particular, quedo de usted.

Atentamente

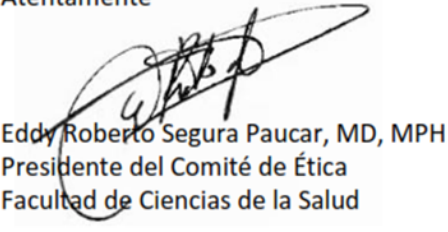




\section{Anexo 2}

\begin{tabular}{|c|c|c|c|}
\hline Score más alto & Score más alto & Score más alto & \\
\hline $\begin{array}{c}\text { ler sextante } \\
(17-14)\end{array}$ & $\begin{array}{c}\text { 2do sextante } \\
\text { (13-23) }\end{array}$ & $\begin{array}{c}\text { 3er sextante } \\
(27-24)\end{array}$ & \\
\hline & & & $\begin{array}{c}\text { Score } \\
\text { acumulativo }\end{array}$ \\
\hline Score más alto & Score más alto & Score más alto & \\
\hline $\begin{array}{c}\text { 6to sextante } \\
(37-34)\end{array}$ & $\begin{array}{c}\text { 5to sextante } \\
(33-43)\end{array}$ & $\begin{array}{c}\text { 4to sextante } \\
(47-44)\end{array}$ & \\
\hline
\end{tabular}




\section{Anexo 3}

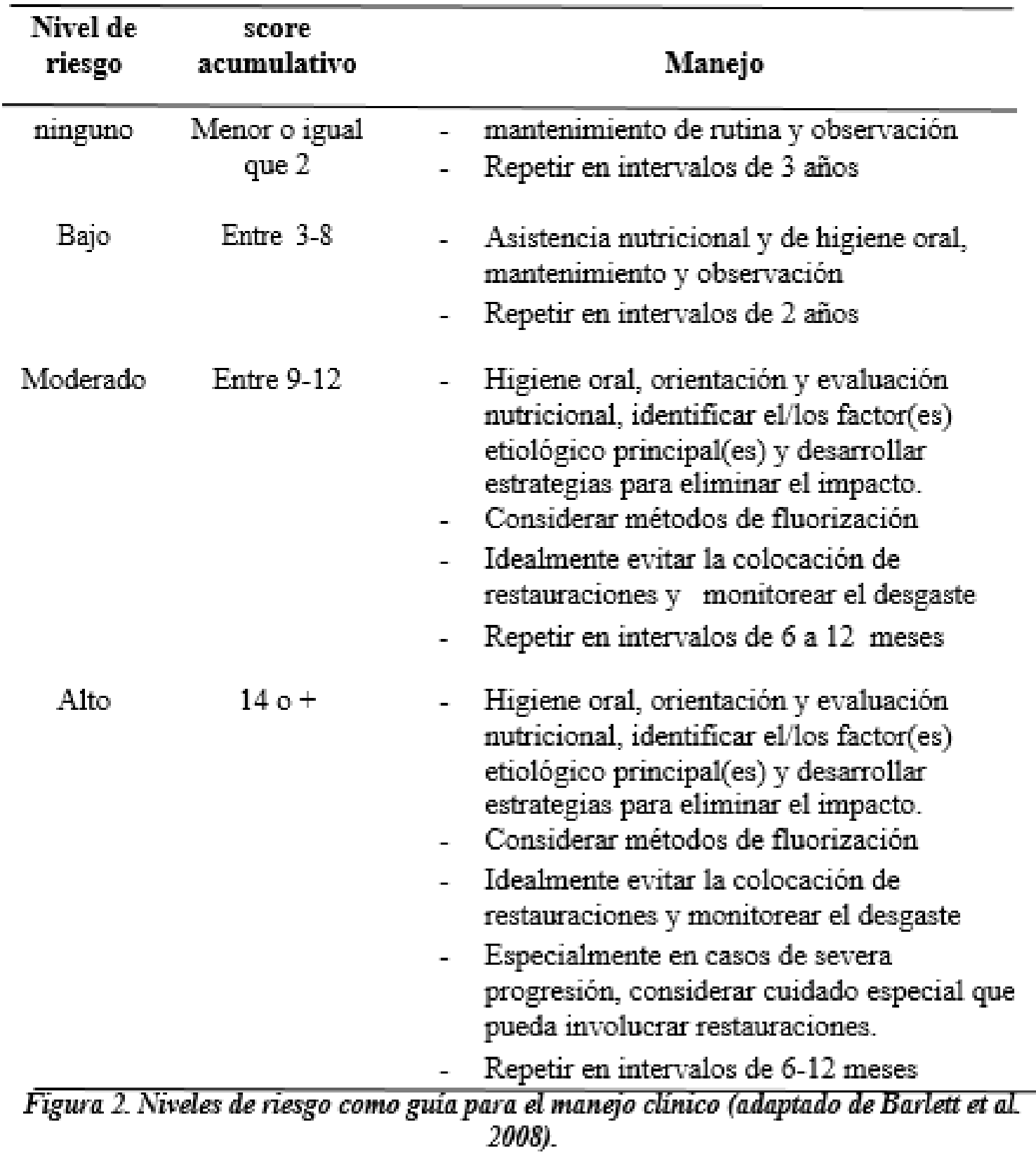

